\title{
Recent Findings on the Psychology of Hydration Habits
}

\author{
Esther Papies Amy Rodger Maria Almudena Claassen Marleen Lomann
}

School of Psychology and Neuroscience, University of Glasgow, Glasgow, UK

\section{Keywords}

Habits · Hydration behavior · Sugar-sweetened beverages ·

Water $\cdot$ Reward

\begin{abstract}
Introduction: This synthesis addresses the psychology of habits in hydration behavior, and presents recent insights about water drinking habits. Habits play a key role in most health behavior, and they allow us to act automatically and without much deliberation or effort. Habits also play a key role in hydration behavior, and are among the strongest predictors of the consumption of water, sugar-sweetened beverages (SSBs), hot beverages, and alcoholic drinks. Methods: We synthesized novel findings from recent in-depth qualitative interviews, a qualitative survey, and a quantitative survey on the role of habits in the consumption of water and sugar-sweetened beverages. Results: Qualitative data show that water drinking is not a simple behavior, and reward plays an important role in water drinking. Participants described numerous barriers to drinking water, including for-
\end{abstract}

getting, lack of access, perceived effort, and others. In addition, our analyses show that water drinking habits are unlikely to emerge or be maintained unless consumers expect experiencing reward from drinking water, for example, because of its taste, cognitive, or physical consequences. Our quantitative study of the effect of the UK pandemic lockdown on drinking behavior points to the effects of reward in drinking habits as well. Situations that typically afford the consumption of SSBs and water outside of the home (e.g., eating out, socializing, parties for SSBs, gym, office, and travel for water) were less frequent during lockdown. Nevertheless, overall consumption of SSBs, but not water, increased in this period, especially among strongly habitual SSB consumers. This was driven by SSB consumption at home, suggesting that participants established new, rewarding consumption habits when their typical consumption situations had disappeared. Conclusion: These findings suggest that experiencing reward from consumption is essential in hydration habits, which may further inform applications to increase healthy hydration.

(c) 2022 The Author(s)

Published by S. Karger AG, Basel karger@karger.com www.karger.com/anm

Karger $\stackrel{\text { ' }}{=}$
(C) 2022 The Author(s)

Published by S. Karger AG, Basel

This article is licensed under the Creative Commons Attribution 4.0 International License (CC BY) (http://www.karger.com/Services/ OpenAccessLicense). Usage, derivative works and distribution are permitted provided that proper credit is given to the author and the original publisher.
Correspondence to:

Esther Papies, esther.papies@glasgow.ac.uk 


\section{Statement of Ethics}

This abstract paper does not present empirical data, but a synthesis of recent findings. In all studies underlying the synthesis presented here, participants have given their written informed consent. All study protocols were approved by the University of Glasgow College of Science and Engineering Ethics Committee (application numbers 300200029, 300190043, 300200055).

\section{Conflict of Interest Statement}

E.P. received travel expenses and registration fee from Danone Research to attend the 2019 Hydration for Health Scientific Conference, for the European Federation of the Associations of Dietitians 2019 Conference, and the 2020 and 2021 European and International Congress on Obesity (online). The University of Glasgow has received speaker fees for E.P. from Danone Research. Danone Research co-funds the ESRC/SGSSS PhD studentship of A.R.

\section{Funding Sources}

The research reported in this presentation was supported by an ESRC/SGSSS studentship to A.R., and by ESRC Research Grant ES/R0055419/1 awarded to E.K.P. The funders had no role in the study design or analysis, or the decision to present these findings.

\section{Author Contributions}

This synthesis was developed by E.K.P., with contributions from A.R., M.A.C., and M.L., who are authors on the original empirical studies synthesized here.

\section{Data Availability Statement}

This abstract paper does not present empirical data. However, 2 of the studies from which findings are synthesized are available online with open access to all underlying data on the Open Science Framework, here https://psyarxiv.com/grndz and here https:// psyarxiv.com $/ \mathrm{kg} 367 /$. 